\title{
Tratamento de crianças com malária pelo Plasmodium falciparum com derivados da artemisinina
}

\author{
Treatment of children with malaria Plasmodiumfaldipanum \\ with derivatives artemisinin
}

\author{
Maria das Graças Costa Alecrim ${ }^{1,2,3}$, Luís Magalhães Carvalho², Solange Dourado Andrade ${ }^{2}$, \\ Ana Ruth Lima Arcanjo ${ }^{2}$, Márcia Araújo Alexandre ${ }^{2}$ e Wilson Duarte Alecrim ${ }^{1,2,4}$
}

\begin{abstract}
Resumo No período compreendido entre janeiro de 1996 e dezembro de 1998, administramos derivados da artemisinina em 108 crianças com malária por Plasmodium falciparum, para avaliar a resposta clínica e terapêutica. Foram incluídas apenas crianças com clínica de malária moderada ou grave. No Grupo I, incluímos 62 pacientes e administramos artesunate por via endovenosa. Clinicamente, 50,8\% tinham malária moderada e 49,2\% malária grave; a parasitemia foi baixa em 53,2\%, média em 22,6\% e alta em 24,2\%; no D2 a parasitemia estava negativa em 58,1\%. No Grupo II, incluímos 46 pacientes que receberam artemeter (Paluter ${ }^{\circledR}$ ) intramuscular. Clinicamente, $67,4 \%$ apresentavam malária moderada e 32,6\% malária grave; a parasitemia foi baixa em 52,2\%, média em 36,2\% e alta em 15,2\%; em D2, 56,5\% apresentaram negativação da parasitemia. Nos dois grupos, a melhora clínica e evolução da parasitemia não mostraram diferença estatística; no D7 havia clareada a parasitemia em todos os pacientes. Para evitar recrudescência usamos mefloquina ou clindamicina.
\end{abstract}

Palavras-chaves: Malária. Plamodium falciparum. Parasitemia. Tratamento. Artemisinina.

Abstract From January 1996 to December 1998, artemisinin derivatives were prescribed to 108 children infected with Plasmodium falciparum. The therapeutic effect was evaluated. Only children with moderate or severe malaria were included. Group I (intravenous artesunate; $n=62$ ): $50.8 \%$ with moderate malaria and 49.2\% with severe malaria; $53.2 \%$ with mild parasitemia, $22.6 \%$ with moderate parasitemia and $24.2 \%$ with high parasitemia; Group II (intramuscular artemether [Paluter ${ }^{\circledR}$ ]; $n=46$ ): $67.4 \%$ with moderate malaria and $32.6 \%$ with severe malaria; $52.2 \%$ with mild parasitemia, $36.2 \%$ with moderate parasitemia and $15.2 \%$ with high parasitemia; clinical amelioration and clearance of parasitemia showed no statistical difference between the groups. All patients cleared the parasitemia at the seventh day of follow-up (D7). In order to avoid recrudescence, mefloquine or clindamycin was used.

Key-words: Malaria. Plasmodium falciparum. Parasitemia. Treatment. Artemisinin.

Malária é a principal endemia da Amazônia brasileira. No Estado do Amazonas, no período compreendido entre janeiro de 1996 a dezembro de 1998, foram diagnosticados 279.914 casos, sendo que o $P$. falciparum contribuiu com 68.021 dos diagnósticos. Na Fundação de Medicina Tropical do Amazonas (FMT/IMT-AM), neste mesmo período, foram diagnosticados e tratados 27.543 pacientes com malária, entre os quais 6.547 eram crianças e destas, 388 foram atendidas em regime de internação, das quais 277 apresentaram malária pelo $P$. falciparum.
Nas áreas de alta endemicidade a mortalidade está concentrada principalmente na população infantil, com evidência de mais de um milhão de óbitos a cada ano. Em crianças africanas, a malária é a principal causa de morbidade e mortalidade; nas formas graves, a malária cerebral chega a $40 \%$ dos óbitos, que podem ocorrer 24 horas após admissão nos hospitais. No Gâmbia, estima-se que $5 \%$ a $10 \%$ das crianças morrem de malária cerebral; na Nigéria a estimativa é de uma em cada três crianças tratadas na Emergência do Hospital

1. Fundação de Medicina Tropical do Amazonas, Manaus, AM. 2. Universidade Federal do Amazonas, Manaus, AM. 3. Centro Universitário Nilton Lins. 4. Universidade do Estado do Amazonas.

Endereço para correspondência: Dra Maria das Graças C. Alecrim. Av. Pedro Teixeira 25, Dom Pedro, 69040-000 Manaus, AM.

Tel: 5592 656-2684, Fax: 5592 238-7220

e-mail:walecrim@uol.com.br

Recebido para publicação em 31/7/2001. 
Universitário tenham malária cerebral. As que sobrevivem ainda podem apresentar seqüelas neurológicas como: hemiplegia, cegueira ou ataxia 4568911.

O tratamento da infecção pelo $P$. falciparum, após o aparecimento de cepas multi-resistentes, ficou mais difícil, sendo necessário o uso de drogas alternativas, mais eficazes para o tratamento da malária nas áreas de resistência ${ }^{1,3}$. Os derivados da artemisinina negativam rapidamente a parasitemia em sangue periférico, são esquizonticidas sangüíneos de ação rápida, entretanto, quando não associados com uma droga de excreção lenta como mefloquina, os pacientes podem apresentar recrudescência. Com o aparecimento das cepas multiresistentes, relatadas principalmente na Tailândia e Brasil, é necessário o uso dos derivados das artemisininas, para o tratamento da malária falciparum. ${ }^{12312} 13$.

Murphy e cols ${ }^{10}$ compararam a eficácia do artemeter IM com o quinino EV em crianças com malária cerebral no Quênia, e não observaram efeitos colaterais significativos entre as duas drogas. A letalidade foi de
$16,2 \%$, não apresentando diferença estatística nos dois grupos, concluíram que na África a resistência ao quinino não é significativa e a administração do artemeter tem como desvantagem a necessidade da associação com outras drogas. White e cols ${ }^{17}$ fizeram um estudo comparativo tratando crianças com malária grave usando artemeter ou cloroquina para avaliar a toxicidade e eficácia das duas drogas, o artemeter clareou mais rapidamente a parasitemia, entretanto não apresentou diferença quanto à evolução para melhora do quadro clínico. Estudos de Looareesuwan e cols ${ }^{8}$ e Sabcharion e cols ${ }^{14}$ avaliaram a eficácia da associação do artesunate com mefloquina em cepas multi-resistentes verificando a negativação da parasitemia nos pacientes estudados.

O objetivo deste trabalho foi de acompanhar a evolução clínica e verificar a eficácia terapêutica entre artesunate endovenoso e artemeter (paluter ${ }^{\circledR}$ ) intramuscular, associados à mefloquina ou à clindamicina, no tratamento de crianças com malária falciparum internadas na FMT/IMT-AM.

\section{PACIENTES E MÉTODOS}

O estudo foi realizado na FMT/ IMT-AM no período de janeiro de 1996 a dezembro de 1998, incluindo uma amostra de 108 crianças com malária falciparum internadas durante oito dias na enfermaria de pediatria. Foi um estudo aberto, e incluímos no protocolo crianças com clínica de malária moderada ou grave. A artemisinina é droga (liberadas pelo Ministério da Saúde do Brasil) prescritas de rotina para o tratamento da malária grave ou com tendência a gravidade na FMT/IMT-AM.

Clinicamente os pacientes foram classificados como portadores de malária moderada ou grave. Com malária moderada os que apresentavam: cefaléia, febre, náuseas, vômitos e calafrio. Malária grave: sinais e sintomas clássicos da malária, já mencionados anteriormente associado a uma ou mais das seguintes complicações: anemia, coma, hipoglicemia, insuficiência renal aguda, coagulação intravascular disseminada, edema agudo de pulmão, plaquetopenia, crise convulsiva, hiperparasitismo ${ }^{18}{ }^{19}$. As crianças foram examinadas diariamente; e a temperatura axilar aferida quatro vezes ao dia. Consideramos o paciente afebril quando a temperatura era igual ou menor que $37^{\circ} \mathrm{C}$, por um período igual ou maior que $24 \mathrm{horas;}$; foi considerada anemia quando a hemoglobina estava igual ou abaixo de $11 \mathrm{~g} / \mathrm{dl}$, plaquetopenia grave, quando a contagem estava igual ou abaixo de 60.000 .

O diagnóstico da malária foi realizado pelo método da gota espessa, com avaliação diária até o sétimo dia (D0 ao D7), e o resultado expresso em trofozoítos por milímetro cúbico de sangue. A parasitemia foi classificada em: baixa, até 15.000 parasitas por $\mathrm{mm}^{3}$ de sangue; média, de 15.001 a 60.000 parasitas por $\mathrm{mm}^{3}$ de sangue e alta, com resultado igual ou maior que 60.001 parasitas por $\mathrm{mm}^{3}$ de sangue ${ }^{1}$.

O esquema posológico usado para o tratamento dos pacientes dependia do quadro clínico e do nível da parasitemia. As crianças foram divididas em dois grupos: no Grupo I incluímos 62 nas quais foi administrado artesunate endovenoso; no Grupo II 46 crianças receberam artemeter (Paluter ${ }^{\circledR}$ ) intramuscular. Artesunate ou artemeter (Paluter $\AA$ ) foram administrados nas doses apresentadas a seguir:

Pacientes com quadro clínico de malária moderada e qualquer nível de parasitemia sem esquizonte no sangue periférico:

Grupo I

Dia 0 - $1^{\text {a }}$ dose - artesunate: $2 \mathrm{mg} / \mathrm{kg} / \mathrm{peso} / \mathrm{dia} / \mathrm{de} 12 / 12$ horas

Dia 1 - 2a dose - artesunate: $1 \mathrm{mg} / \mathrm{kg} / \mathrm{peso} / \mathrm{dia} / \mathrm{de} 12 / 12$ horas

Grupo /I

Dia 0 - $1^{\text {a }}$ dose - artemeter (Paluter ${ }^{\circledR}$ ) 2mg/kg/peso/dia/de 12/12 horas

Dia 1 - 2a dose - artemeter (Paluter $\left.{ }^{\circledR}\right)$ 1mg/kg/peso/dia/de 12/12 horas

Pacientes com malária clinicamente grave e/ou parasitemia com esquizonte em sangue periférico:

Grupo I

Dia 0 - $1^{\text {a }}$ dose artesunate $-2 \mathrm{mg} / \mathrm{kg} / \mathrm{peso} / \mathrm{dia}$, dividido em duas doses. A segunda dose foi prescrita seis horas após a primeira

Dia 1 - $3^{\text {a }}$ dose artesunate $-1 \mathrm{mg} / \mathrm{kg} / \mathrm{peso} / \mathrm{dia} / \mathrm{de}$ 12/12 horas, prescrita 24 horas após a primeira dose.

Grupo /I

Dia 0 - $1^{\text {a }}$ dose - artemeter (Paluter $\left.{ }^{\circledR}\right) 2 \mathrm{mg} / \mathrm{kg} / \mathrm{peso} /$ dia dividido em duas doses. A segunda dose foi prescrita seis horas após a primeira

Dia 1 - $3^{\text {a }}$ dose artemeter (Paluter $\left.{ }^{\circledR}\right) 1 \mathrm{mg} / \mathrm{kg} /$ peso/dia de 12/12 horas, prescrita 24 horas após a primeira dose.

Nos dois grupos, em D2, foi usada a associação com mefloquina na dosagem de $20 \mathrm{mg} / \mathrm{kg} /$ peso dividida em duas tomadas, com intervalo de seis horas, ou clindamicina quando havia contra-indicação da mefloquina, na dosagem de $20 \mathrm{mg} / \mathrm{kg} /$ peso de $12 \mathrm{em}$ 12 horas, durante cinco dias. 
$\mathrm{Na}$ internação foram realizados exames laboratoriais como: hemograma e contagem de plaquetas, dosagem de alanina aminotransferase (ALT) e aspartato aminotransferase (AST), bilirrubinas, glicose, uréia e creatinina.

\section{RESULTADOS}

No Grupo I fizeram parte 62 crianças. Destas, 37 (59,7\%) eram do sexo masculino e $25(40,3 \%)$ do sexo feminino, tratadas com artesunate endovenoso; em $57(91,9 \%)$ associamos mefloquina, e em 5 (8,1\%) clindamicina. Quanto à faixa etária, $20(32,2 \%)$ pertenciam ao grupo etário de 0 2 anos, $16(25,8 \%)$ de $3-5$ anos, $16(25,8 \%)$ de $6-8$ anos e $10(16,2 \%)$ acima de oito anos. Segundo a procedência, $58(93,5 \%)$ eram natural do Amazonas, $2(3,3 \%)$ do Pará, 1 $(1,6 \%)$ de Roraima e $1(1,6 \%)$ ignorada. A classificação clínica mostrou que $32(50,8 \%)$ tinham malária moderada e 31 $(49,2)$ malária grave. Na classificação da densidade parasitária, $33(53,2 \%)$ tinham baixa parasitemia, $14(22,6 \%)$ média parasitemia e $15(24,2 \%)$ alta parasitemia. A manifestação clínica encontrada com maior freqüência foi a febre; no momento da internação, todos os pacientes apresentavam temperatura acima de $38^{\circ} \mathrm{C}$.

A principal complicação foi anemia em 57 (91,9\%) crianças. Anemia grave, com hemoglobina igual ou abaixo de $5 \mathrm{~g} / \mathrm{dl}$, esteve presente em $2(3,2 \%)$ pacientes. Plaquetopenia foi observada em $30(48,4 \%)$ doentes e destes, $2(3,2 \%)$ apresentaram plaquetas abaixo de 10.000 (plaquetopenia grave). Malária cerebral ocorreu em $3(4,8 \%)$. Icterícia em 10 (16,1\%). Insuficiência renal em $1(1,6 \%)$, hipoglicemia em $1(1,6 \%)$ e crise convulsiva em $1(1,6 \%)$. Quanto à negativação da parasitemia verificamos em D2 $59,4 \%$ dos doentes com quadro de malária moderado com lâmina negativa; com malária grave $56,7 \%$ (Tabela 1).

No Grupo II, incluímos 46 crianças que foram tratadas com artemeter (Paluter $\left.{ }^{\circledR}\right)$ intramuscular, associada à mefloquina em $43(93,4 \%)$ e nos demais à clindamicina; $25(54,4 \%)$ pertenciam ao sexo masculino e $21(45,6 \%)$ ao sexo feminino. $\mathrm{Na}$ distribuição por faixa etária, $20(43,5 \%)$ pertenciam ao grupo etário de $0-2$ anos, $10(21,7 \%)$ de $3-5$ anos, $8(21,7 \%)$ de $6-8$ anos e $8(21,7 \%)$ ao de acima de oito anos. Segundo a procedência, $43(93,5 \%)$ eram do Amazonas e $3(6,5 \%)$ ignorada. A classificação clínica mostrou $31(67,4 \%)$ com malária moderada e $15(32,6 \%)$ com malária grave. Na classificação da densidade parasitária $24(52,2 \%)$ tinham baixa parasitemia, $15(32,6 \%)$ média parasitemia e 7 $(15,2 \%)$ alta parasitemia. A principal manifestação clínica encontrada foi a febre; no momento da internação todos os pacientes apresentaram temperatura acima de $38^{\circ} \mathrm{C}$, seguida de calafrios, vômitos e mialgia.

A principal complicação foi anemia em 44 (95,7\%) crianças. Destas $1(2,2 \%)$, apresentou anemia grave com hemoglobina de $5 \mathrm{~g} / \mathrm{dl}$. Plaquetopenia foi observada em 24 $(52,2 \%)$ pacientes e destes $1(2,1 \%)$ apresentou plaquetas abaixo de 10.000 (plaquetopenia grave). Malária cerebral estava presente em $3(4,8 \%)$. Icterícia em $10(16,1 \%)$. Hipoglicemia em $2(4,3 \%)$ e crise convulsiva $2(4,3 \%)$. Quanto à negativação da parasitemia, verificamos que em D2 $64,5 \%$ dos doentes com quadro de malária moderada estavam com lâmina negativa; com malária grave $40 \%$ (Tabela 1).

Tabela 1 - Evolução da parasitemia nas 108 crianças com malária por P. falciparum tratadas com artesunate ou artemeter (Paluter ${ }^{\circledR}$ ).

\begin{tabular}{|c|c|c|c|c|c|c|c|c|}
\hline \multirow[b]{3}{*}{ Dia } & \multicolumn{4}{|c|}{ Moderada } & \multicolumn{4}{|c|}{ Grave } \\
\hline & \multicolumn{2}{|c|}{$\begin{array}{c}\text { artesunate } \\
\text { grupo I }\end{array}$} & \multicolumn{2}{|c|}{$\begin{array}{c}\text { artemeter } \\
\text { grupo II }\end{array}$} & \multicolumn{2}{|c|}{$\begin{array}{c}\text { artesunate } \\
\text { grupo I }\end{array}$} & \multicolumn{2}{|c|}{$\begin{array}{c}\text { artemeter } \\
\text { grupo II }\end{array}$} \\
\hline & $\mathrm{n}^{\circ}$ & $\%$ & $\mathrm{n}^{\circ}$ & $\%$ & $\mathrm{n}^{\circ}$ & $\%$ & $\mathrm{n}^{\circ}$ & $\%$ \\
\hline D0 & 32 & 100,0 & 31 & 100,0 & 30 & 100,0 & 15 & 100,0 \\
\hline D1 & 6 & 18,8 & 5 & 16,1 & 2 & 6,7 & 2 & 13,3 \\
\hline D2 & 19 & 59,4 & 20 & 64,5 & 17 & 56,7 & 6 & 40,0 \\
\hline D3 & 5 & 15,6 & 6 & 19,4 & 9 & 30,0 & 4 & 26,7 \\
\hline D4 & 1 & 3,1 & neg & neg & 1 & 3,3 & 2 & 13,3 \\
\hline D5 & 1 & 3,1 & neg & neg & 1 & 3,3 & 1 & 6,7 \\
\hline D6 & neg & neg & neg & neg & neg & neg & neg & neg \\
\hline D7 & neg & neg & neg & neg & neg & neg & neg & neg \\
\hline Total & 32 & 100,0 & 31 & 100,0 & 30 & 100,0 & 15 & 100,0 \\
\hline
\end{tabular}

\section{DISCUSSÃO}

As manifestações clínicas da malária grave são várias ${ }^{510}$ e estã̃o geralmente relacionadas com o $P$. falciparum, incluindo anemia, acidose metabólica, choque, distúrbios da coagulação, edema agudo de pulmão ou comprometimento cerebral. Os derivados das artemisininas negativam rapidamente a parasitemia, sendo atualmente as drogas de escolha para o tratamento da malária grave e de cepas multi-resistentes. Taylor e cols ${ }^{15}$ usaram artemeter ou quinino no tratamento de crianças com malária cerebral, verificando que a 
melhora clínica foi significativamente mais rápida na primeira droga. Outros autores têm avaliado a eficácia do uso das artemisininas na malária grave ${ }^{16}{ }^{17}$.

Neste estudo, tratamos crianças apresentando malária grave ou moderada com artesunate endovenoso ou artemeter (Paluter $\left.{ }^{\circledR}\right)$ intramuscular, associado à mefloquina ou clindamicina para verificar a eficácia das duas drogas na evolução clínica e negativação da parasitemia. $\mathrm{Na}$ avaliação clínica das 63 crianças com malária moderada e das 45 crianças com malária grave, $69,4 \%$ dos pacientes tratados com artesunate e $76,1 \%$ artemeter (Paluter ${ }^{\circledR}$ ) ficaram apiréticos em D2, mostrando a eficácia dos derivados das artemisininas na melhora clínica desses pacientes.

A principal complicação encontrada foi anemia em 67 crianças com hemoglobina igual ou abaixo de $11 \mathrm{~g} / \mathrm{dl}$, mostrando que a anemia, também causada por vários processos, torna-se mais importante nas crianças, com malária, procedentes da Amazônia brasileira. As manifestações da malária cerebral foram diagnosticadas em cinco crianças, nenhuma evoluiu para óbito, mostrando que o diagnóstico precoce associado ao tratamento adequado, ou seja às drogas esquizonticidas de ação rápida, reduzem a letalidade.

Quanto à negativação da parasitemia os pacientes com malária moderada medicados com artesunate em D2, $59,4 \%$ apresentaram lâmina negativa. Nas crianças que usaram artemeter (Paluter $\AA$ ), $64,5 \%$ estavam negativas; nas com malária grave a negativação foi de $56,7 \%$ para o artesunate e $40 \%$ artemeter (Paluter ${ }^{\circledR}$ ), mostrando melhora clínica.

Concluímos em oito dias de acompanhamento, que crianças com malária moderada e grave tratadas com artesunate endovenoso ou artemeter (Paluter ${ }^{\circledR}$ ) intramuscular, associados a mefloquina ou à clindamicina apresentaram a mesma evolução clínica e parasitológica.

\section{REFERÊNCIAS BIBLIOGRÁFICAS}

1. Alecrim MGC. Estudo da Resistência do P. falciparum in vitro e in vivo na Amazônia brasileira. Tese de Mestrado, Universidade de Brasília, Brasília, DF,1981.

2. Alecrim WD, Silva EB, Silva VA, Alecrim MGC. Tratamento da malária $P$. falciparum com a associação de artemeter mais mefloquina. Revista da Sociedade Brasileira de Medicina Tropical 28(sup I):160, 1995.

3. Alecrim MGC, Carvalho LM, Fernandes MC, Andrade SD, Loureiro AC, Arcanjo AR, Alecrim WD. Tratamento da malária com artesunate (retocaps ${ }^{\circledR}$ ) em crianças da Amazônia brasileira. Revista da Sociedade Brasileira de Medicina Tropical 33:163-168, 2000.

4. Cardoso MA, Ferreira MU, Camargo LMA, Szarfare SC. Anaemia, iron deficiency and malaria in a rural community in Brazilian Amazon. European Journal of Clinical Nutrition 48: 326-332, 1994.

5. English M, Waruiru C, Marsh K. Transfusion for respiratory distress in life-threatening childhood malaria. American Journal Tropical Medicine and Hygiene 55: 525-530, 1996.

6. Falade CO, Salako LA, Sowunmi A, Oduola AMJ, Larcier P. Comparative efficacy of halofantrine, chloroquine and sulfadoxinepyrimethamine for treatment of acute uncomplicated falciparum malaria in Nigerian children. Transactions of the Royal Society of Tropical Medicine and Hygiene 91: 58-62, 1997.

7. Hien TT, Arnold K, Hung NT, Loc PP, Dung NT, Cuong BM, Toan LM, Phung MQ, Anh LHV, Mai PP. Single dose artemisinin-mefloquine treatment for acute uncomplicated falciparum malaria. Transactions of the Royal Society of Tropical Medicine and Hygiene 88: 688-691, 1994.

8. Looareesuwan S, Wilairatana P, Molunto W, Chalermrut K, Olliaro $\mathrm{P}$, Andrial M. A comparative clinical trial of sequential treatments of severe malaria with artesunate suppository followed by mefloquine in Thailand. American Journal Tropical Medicine and Hygiene 57: 348-353, 1997.

9. Mendis KN, Carter R. Clinical Disease and Pathogenesis in Malaria. Parasitology Today 11: 2-15, 1995.

10. Murphy S, English M, Waruiru C, Mwangi I, Amukoye E, Crawley J, Newton C, Winstanley P, Peshu N, Marsh K. An open randomized trial of artemether versus quinine in the treatment of cerebral malaria in African children. Transactions of the Royal Society of Tropical Medicine and Hygiene 90: 298-301, 1996.

11. Newton CRJC, Chokwe T, Schellenberg JA, Forster D, Peshu N, Kirkham FJ, Marsh K. Coma scales for children with severe falciparum malaria. Transactions of the Royal Society of Tropical Medicine and Hygiene 91: 161-165, 1997.

12. Noronha EF. Estudo clinico epidemiológico da malária falciparum em crianças de 0 a 14 anos atendidas, no Instituto de Medicina Tropical do Amazonas, em Manaus. Tese de Mestrado, Universidade de Brasília, Brasília,DF, 1998.

13. Ronn AM, Msangeni HA, Mhina J, Wernsdorfer WH, Bygbjerg IC. High level of resistance of Plasmodium falciparum to sulfadoxinepyrimethamine in children in Tanzania. Transactions of the Royal Society of Tropical Medicine and Hygiene 90: 179-181, 1996.

14. Sabcharion A, Attanath $P$, Chanthavanich $P$, Phanuaksook $P$, Prarinyanupharb V, Poonpanich Y, Mookmanee D, TejaIsavadharm P, Heppner DG, Brewer TG, Chongsuphajaisiddhi T. Comparative clinical trial of artesunate suppositories and oral artesunate in combination with mefloquine in the treatment of children with acute falciparum malaria. American Journal Tropical Medicine and Hygiene 58: 11-16, 1998.

15. Taylor T, Wills B, Kazenibe P, Chisale M, Wirima J, Ratsma E, MolyNeux M. Rapid coma resolution with artemeter in Malawian children with cerebral malaria. The Lancet 341:661-662, 1993.

16. Walker P, Salako LA, Omokhodion SI, Sowunmi A. An open randomized comparative study of intramuscular artemether and intravenous quinine in cerebral malaria in children. Transactions of the Royal Society of Tropical Medicine and Hygiene 87: 564-566, 1993.

17. White NJ, Waller D, Jane C, Nosten F, Chapman D, Brewster D, Greenwood. Comparison of artemether and chloroquine for severe malaria in Gambian children. The Lancet 339: 317-321, 1992.

18. World Health Organization. Severe falciparum malaria. Transactions of the Royal Society of Tropical Medicine and Hygiene 94 (supl I), 2000. 